\title{
Toxic encephalopathy associated with low dose metronidazole therapy in a cat suffering from gastrointestinal lymphoma: A case report
}

\author{
Miłosława Kwiatkowska, Urszula Pasławska, Jędrzej M. Jaśkowski \\ Department of Clinical Sciences and Diagnostic, Institute of Veterinary Medicine, \\ Faculty of Biological and Veterinary Sciences, Nicolaus Copernicus University, \\ Gagarina 7, 87-100 Toruń, Poland. \\ kwiatkowska.miloslawa@umk.pl; https://orcid.org/0000-0001-6119-6885, \\ urszula.paslawska@umk.pl; https://orcid.org/0000-0003-2298-9713, \\ jmjaskowski@umk.pl,https://orcid.org/0000-0002-4676-7990
}

Corresponding author: dr Miłosława Kwiatkowski, Department of Clinical Sciences and Diagnostic, Institute of Veterinary Medicine, Faculty of Biological and Veterinary Sciences, Nicolaus Copernicus University, Gagarina 7, 87-100 Toruń, Poland.

E-mail: kwiatkowska.miloslawa@gmail.com 


\section{Abstract}

This case report describes an episode of acute encephalopathy in a cat treated with metronidazole due to chronic gastrointestinal disease (giardiasis). Metronidazole had been administered at a dose of $28 \mathrm{mg} / \mathrm{kg} /$ day for 7 days. Cat showed signs of head pressing, reduced consciousness, ataxia, seizures, lack of menace response and progressive weakness. Complete blood cell count revealed haemolytic anaemia, serum biochemistry, coagulation profile, blood pressure measurement, urinalysis, were unremarkable. Metronidazole administration was immediately discontinued; supportive care consisted of fluid therapy, blood transfusion was also performed. The neurological status of the patient improved rapidly within $72 \mathrm{~h}$. Anaemia improved in next 7 days, however transfusion and steroid treatment was necessary. Two weeks post intoxication abdominal ultrasound revealed abnormal hepatic pattern, enlarged mesenteric lymph nodes and lack of normal intestinal layering. A biopsy had been taken during explorative laparotomy and histopathologic examination revealed gastrointestinal and hepatic lymphoma. The aim of this case report is to present that cats suffering from multiple conditions may show metronidazole intoxication even when recommended dosage and treatment length is used. We cannot exclude that metronidazole was responsible for autoimmune haemolytic anaemia.

Keywords: metronidazole toxicity; cat; lymphoma, liver; gastrointestinal tract.

\section{Introduction}

Metronidazole is a commonly used drug in veterinary medicine, most commonly to treat a wide variety of conditions, including inflammatory bowel disease (IBD) in both dogs and cats, protozoal infections (e.g., giardiasis), bacterial infection associated gastritis, and hepatoencephalopathy. Metronidazole has been also reported to have immunomodulatory effect. The drug is lipophilic and has a wide tissue distribution, it's oral bioavailability ranges from 50 to $100 \%$. Metronidazole is primarily metabolised in the liver and crosses the blood-brain barrier rapidly, is renally excreted, and has a half-life of approximately eight hours. Metronidazole crosses brain-blood barrier, thus central nervous system (CNS) side effects have been reported in humans and in veterinary species, including rats, dogs and cats. Most commonly visible signs are vestibular ataxia, 
nystagmus, head tilt weakness, nausea and abnormal mentation, seizures. Peripheral neuropathies have been reported. Other adverse effects include neutropenia, hepatotoxicity, haematuria, vomiting and diarrhoea. Various sources state that the metronidazole dose in canine and feline patients for anaerobic infectious and gastrointestinal conditions is $10-15 \mathrm{mg} / \mathrm{kg}$ every $12 \mathrm{~h}$ and the dose for protozoal infections is as high as $25 \mathrm{mg} / \mathrm{kg}$ every12 $\mathrm{h}$ for 5 days. The recommended dosage for long-term treatment in dogs is $10 \mathrm{mg} / \mathrm{kg}$ p.o. twice or three times a day. To our knowledge, this is the first report to document CNS signs and autoimmune haemolytic anaemia associated with metronidazole administration at dosage not excessing $30 \mathrm{mg} / \mathrm{kg} /$ day. We conclude that coexisting, silent conditions, hepatic and intestinal lymphoma has altered its metabolism.

\section{Case description}

A seven year old main coon was presented due to two month history of recurrent diarrhoea, waxing and waning appetite and weight loss. Physical examination was unremarkable. A diagnostic plan included laboratory examination (haematology, biochemistry, urinalysis, routine faecal flotation) and diagnostic imaging (abdominal ultrasound). A complete blood cell count and serum biochemistry revealed mild lymphopenia, neutrophilia without a left shift, mild hypoalbuminemia, mild hyperglycaemia, hypophosphatemia and mild elevation of alanine aminotransferase (ALT). Urinalysis and abdominal ultrasound were unremarkable. Faecal sample revealed presence of Giardia intestinalis, further the microorganism has been confirmed with immunofluorescence assay (IFA).

Cat has been started on metronidazole treatment at a dose of $28 \mathrm{mg} / \mathrm{kg} /$ day divided into two dosages. Seven days post initiating treatment with metronidazole cat represented to veterinary clinic due to signs of obtundation and head pressing consistent with forebrain dysfunction. Blood examination (haematology and biochemistry) was performed, results were unremarkable. Within next 12 hours signs progressed to head tilt, generalised ataxia, lack of menace response, cat became very weak 
and was hypersalivating. Blood examination has been repeated and revealed acute, autoimmune haemolytic anaemia (AIHA) with haematocrit of $20 \%$. Autoagglutination test was positive. Due to clinical signs and unremarkable clinical investigations metronidazole metronidazole intoxication was suspected. After the immediate discontinuation of the metronidazole treatment, the patient improved greatly during the first three days with supportive care (acetated Ringer's solution intravenously through a cephalic catheter, assisted feeding, monitoring of vital signs). Due to AIHA a blood transfusion has been performed and cat has been started on steroid treatment. Three days later haematocrit has improved and value of $33 \%$ has been obtained. Due to clinical improvement cat has been discharged and sent home. One week later during a recheck visit blood examination was unremarkable. Due to chronic and recurrent diarrhoea recheck abdominal ultrasound was performed and revealed enlarged mesenteric lymph nodes, with the largest being $3,2 \mathrm{~cm}$ by $0,7 \mathrm{~cm}$ and were ununiformly echoic. Intestinal layering was abnormal. Liver had ununiform echogenicity, a mottled heterogenous appearance with loss of lobular pattern may was seen. A laparoscopic biopsy of lymph nodes and intestines has been performed and histopathologic examinations revealed lymphoma.

\section{Discussion and conclusions}

Metronidazole is widely used in clinical practice, the neurotoxicity of metronidazole seems to be under recognized $[1,2]$. When patients are started on this antimicrobial they are not always informed about the potential neurologic complications ${ }^{3}$. Fortunately, even if side effects appear, they are often fully reversible after drug is discontinued. Side effects may also be less obvious in complicated patients due to polypharmacy or due to coexisting conditions predisposing them to neurological diseases [3]. The presented case report depicts the risks of a middle-term (one week) and low dosage therapy in cats. The symptoms detected in our patient revealed a vestibulocerebellar disorder and forebrain disorder, this type 
and distribution of the CNS lesions are consistent with both natural and experimental cases of metronidazole toxicosis previously reported in veterinary medicine [3-6]. Surprisingly in our case metronidazole dosage that caused neurologic signs was $(28 \mathrm{mg} / \mathrm{kg} /$ day) below previously reported toxic dosage $(30 \mathrm{mg} / \mathrm{kg} /$ day), also the treatment time was quite short (7 days). Metronidazole neurotoxicity has been previously reported in 5 cats [7-8] In other report of reversible CNS toxicosis associated with metronidazole therapy in three cats, one cat received $48 \mathrm{mg} / \mathrm{kg} / \mathrm{d}$ for 10 months prior to presentation, in the second cat was $62.5 \mathrm{mg} / \mathrm{kg} / \mathrm{d}$ for $1 \mathrm{month}$, and the third cat received $62 \mathrm{mg} / \mathrm{kg} / \mathrm{d}$ for 5 days [7-8] Thee dosages were much higher than one reported by us.

We suspect that one the reasons why out patent suffered from metronidazole toxicity despite quite low dosage might have been the fact that cat suffered from intestinal lymphoma. Based on the ultrasound image, and post mortem histopathologic examination liver was affected by lymphoma either [9]. We conclude that severity of neurological signs might have been influenced by altered metronidazole absorption in gastrointestinal tract, and altered metabolism in liver.

Gastrointestinal type of lymphoma accounts for $50-70 \%$ of feline lymphoma cases. It is most common in senior cats, with the average age at diagnosis ranging from 9-13 years old [9-10]. Hepatic involvement is associated with a variable appearance. In some cases, the liver appears to be grossly normal. In others, a uniformly enhanced lobular pattern, or a mottled heterogenous appearance with loss of lobular pattern may be seen. With focal mass lesions, multifocal or solitary raised areas of variable colour may be observed.

An association between lymphoma and feline immunodeficiency virus (FIV) has also been proposed. Cats infected with FIV, FeLV, or both FIV and FeLV were determined to have 5.6, 62.1, and 77.3 times the relative risk of developing lymphoma, respectively [9-10]. Our patient has been FIV, FeLV and FIV negative in commercially available tests. Therefore we think that infectious diseases had no influence on neurologic signs.

The pathophysiology underlying metronidazole neurotoxicity remains unclear although several possible mechanisms have been proposed. 
It is not known whether metronidazole itself is neurotoxic or whether it Is of its metabolites. Metronidazole has been shown to bind to RNA and therefore may inhibit protein synthesis further leading to axonal degeneration [11-12]. Another suggested mechanism is its role in free radical formation. Metronidazole's structure is similar to the thiazole, which is precursor of thiamine, it has been suggested that intestinal flora possessing thiaminase activity may be involved in the conversion of metronidazole to a thiamine antagonist, further leading to neurotoxicity [13]. Although there are no consistent patient-related factors, some have suggested that advanced age as well as renal and hepatic dysfunction may confer a higher risk of developing metronidazole-related side effects. Most commonly reported adverse effects include neutropenia, hepatotoxicity, haematuria, vomiting and diarrhoea, but In literature there has been no data regarding anaemia that might have been associated with metronidazole treatment so far [14-15].

Previous case definitions of metronidazole toxicosis in veterinary species have been based on a history of having received metronidazole, showing compatible clinical signs, having an absence of other clinical disease, and experiencing the eventual recovery upon discontinuation of the drug. Despite the fact that no diagnostic imaging e.g. magnetic resonance imaging [16] or computed tomography had been performed, on the basis of above criteria-diagnosis of metronidazole neurotoxicosis had been made $[3,17]$.

\section{References}

[1] Volkmann M, Steiner JM, Fosgate GT et al.Chronic diarrhoea in dogs: retrospective study in 136 cases. J Vet Intern Med. 2017;3:1043-1055.

[2] Tangtrongsup S, Scorza V. Update on the diagnosis and management of Giardia spp infections in dogs and cats. Top Companion Anim Med. 2010; 25:155-162.

[3] Gupta AK, Agarwal MP, Avasthi R, Bhadoria DP, et al. Metronidazole-induced neurotoxicity. J Assoc Physicians India. 2000; 51:617-618. 
[4] Halloran TJ. Convulsions associated with high cumulative doses of metronidazole. Drug Intell Clin Pharm. 1982;16:409.

[5] Agarwal A, Kanekar S, Sabat S, Thamburaj K. Metronidazole-induced cerebellar toxicity. Neurol Intern. 2016;8:63-65.

[6] Hajek I, Simerdova V, Vavra M et al.Toxic encephalopathy associated with high-dose metronidazole therapy in a dog: a case report. Vet Med. 2017;62:105-110.

[7] Saxon, B, Magne, ML: Reversible central nervous system toxicosis associated with metronidazole therapy in three cats. Prog Vet Neurol. 1993;4:25-27.

[8] Caylor, KB, Cassimatis, MK: Metronidazole neurotoxicosis in two cats. J Am Anim Hosp Assoc. 2001;37:258-262

[9] Richter KP. Feline gastrointestinal lymphoma Vet Clin North Am Small Anim Pract. 2003; 33:1083-1098.

[10] Gieger T. Alimentary lymphoma in cats and dogs. Vet Clin North Am Small Anim Pract. 2011;41:419-432.

[11] Kusumi RK, Plouffe JF, Wyatt RH et al. Central nervous system toxicity associated with metronidazole therapy. Ann Intern Med. 1980;9:59-60.

[12] Olson EJ, Morales SC, McVey AS, Hayden DW. Putative Metronidazole Neurotoxicosis in a Cat. Vet Pathol. 2005;42:665-669.

[13] Hajek I, Simerdova V, Vavra M, Agudelo CF. Toxic encephalopathy associated with high-dose metronidazole therapy in a dog: a case report. Veterinarni Medicina. 2017;62:105-110.

[14] Dow SW, LeCouteur RA, Poss ML, Beadleston D. Central nervous system toxicosis is associated with metronidazole treatment of dogs: five cases (1984-1987). Journal of the American Veterinary Medical Assoc. 1989;195:365-368.

[15] Nau R, Sorgel F, Eiffert H. Penetration of drugs through the blood-cerebrospinal fluid/blood-brain barrier for treatment of central nervous system infections. Clinical Microbiology Rev. 2010;23, 858-883.

[16] Miljkovic V, Arsic B, Bojanic Z et al.Interactions of metronidazole with other medicines: a brief review. Pharmazie. 2014;6:571-577.

[17] Ahmed A, Loes DJ, Bressler EL. Reversible magnetic resonance imaging findings in metronidazole-induced encephalopathy. Neurology. 1995;45:588589. 